\section{$\underset{\substack{\text { hommes } \\ \text { \& migrations }}}{ }$}

\section{Hommes \& migrations}

Revue française de référence sur les dynamiques

migratoires

$1313 \mid 2016$

1983, le tournant médiatique

\title{
Les 40 ans du Centre Mandapa
}

\section{François Bensignor}

\section{OpenEdition \\ Journals}

\section{Édition électronique}

URL : http://journals.openedition.org/hommesmigrations/3597

DOI : 10.4000/hommesmigrations.3597

ISSN : 2262-3353

\section{Éditeur}

Musée national de l'histoire de l'immigration

\section{Édition imprimée}

Date de publication : 1 janvier 2016

Pagination : 160-164

ISBN : 978-2-919040-34-6

ISSN : $1142-852 X$

\section{Référence électronique}

François Bensignor, «Les 40 ans du Centre Mandapa », Hommes \& migrations [En ligne], 1313 | 2016, mis en ligne le 17 juin 2016, consulté le 15 septembre 2020. URL : http://journals.openedition.org/ hommesmigrations/3597 


\section{MUSIQUES}

\section{LES 40 ANS DU CENTRE MANDAPA}

par FRANÇOIS BENSIGNOR.

$E^{n}$ n 1975, naissait le Centre Mandapa, au cœur du XIII arrondissement de Paris. D'abord dédié à la danse indienne, ce lieu pionnier est l'une des rares scènes de la capitale présentant régulièrement des spectacles de musiques et danses du monde. Milena Salvini, qui l'a fondé avec son mari et le dirige, a joué un rôle primordial pour une meilleure connaissance des formes de représentations musicales, théâtrales et dansées de I'Inde en France et dans le monde. La relation privilégiée qu'elle entretient avec Ariane Mnouchkine a donné naissance à des nuits mémorables au Théâtre du Soleil, notamment consacrées aux à l'art du Kathakali et du Kûtiyattam.

Née à Milan d'un père italien et d'une mère française, Milena Salvini est âgée de quatre ans lorsque sa mère s'installe à Paris après le décès de son mari. Pianiste, celle-ci évolue dans l'univers des bals, des piano-bars et des cabarets de chanson. Pour la petite Milena, la voie est toute tracée : solfège, piano, puis, à 15 ans, accession au Conservatoire national de musique. Brillante élève, ses études s'achèvent en 1955 avec deux premiers prix, en contrepoint et en harmonie.

La jeune Milena est alors animée par deux passions : "J'ai hésité, dans ma jeunesse à me diriger vers la danse ou la musique, raconte-t-elle. Parallèlement à la musique, j'ai étudié la danse classique, ainsi que le mime. "Sa découverte, grâce au Théâtre des Nations, des spectacle de danse indienne qui incluent la musique et le jeu théâtral codé, orientera sa destinée. Entre 1957 et 1968, le Théâtre des Nations (ancien Sarah Bernhardt) présentait des auteurs et compositeurs du monde entier : Bertolt Brecht, Jerzy Grotowski, Maurice Béjart, Julian Beck, Alban Berg, etc. Mais aussi des formes traditionnelles et rituelles peu connues en Occident, comme l'opéra chinois, le Nô japonais ou le Bharata Natyam indien. " On avait l'occasion pour la première fois - c'était un événement dans l'histoire du théâtre - de voir des troupes traditionnelles venant de différents pays du monde. Découvrir la danse indienne a été pour moi un moteur. Voulant m'y intéresser de près, j'ai demandé une bourse et suis partie étudier en Inde. " De la fin 1963 à la fin 1965, Milena Salvini approche plusieurs formes de danse et décide de se spécialiser dans l'étude du Kathakali au Kerala. En 1964, elle est la première Française admise à l'école du Kalamandalam et étudie auprès du maître K. Padmanabhan Nair (1927-2007).

"En Inde, les disciplines artistiques de tradition orale se transmettent de maître à disciple, de père en fils, en petite communauté, explique la danseuse. Souvent le maitre a un disciple privilégié et quelques autres. Je suis restée très attachée à l'école de mon maitre. Mon engagement moral était aussi de continuer à travailler avec ses disciples et de faire connaitre cette institution. " En 


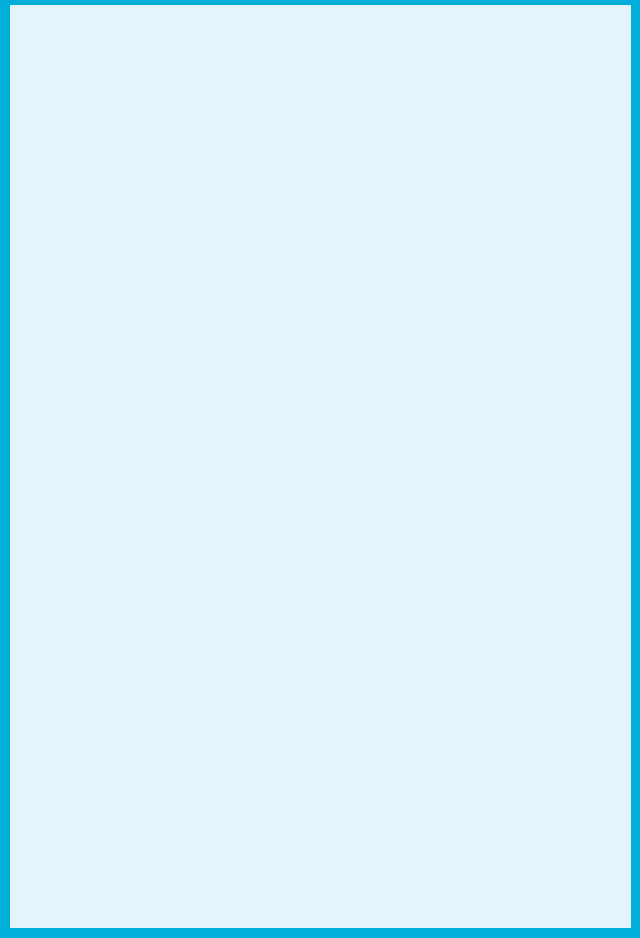

Kathakali, photos extraites du film de M. Salvini et R. Filipuzzi, réalisé par Jacques Oger, L'Épopée du Mahabharata, tourné en décors naturels $\odot$ D.R.

1965, la direction du Théâtre des Nations avait été confiée à Jean-Louis Barrault. Dès son retour en France, Milena Salvini approche le metteur en scène et le convainc de faire venir la troupe du Kalamandalam constituée d'une vingtaine d'artistes. Repartie en Inde, elle y approfondit son étude de la discipline, tout en travaillant avec les artistes à l'élaboration d'un spectacle pouvant être présenté à l'international selon les critères occidentaux. " J'ai collaboré étroitement à la sélection des artistes et des pièces du répertoire extraites essentiellement du Ramayana et du Mahabharata, » explique-t-elle. Le grand spectacle de Kathakali qui en résulte est présenté au Théâtre de l'Odéon en 1967, avant d'entamer une tournée de quatre mois et demi, qui le conduit autour du monde jusqu'à Broadway, en passant par Berlin, l'Italie ou de grands festivals comme celui de Balbek.

\section{Les modes de représentation}

"Au XVII siècle, le Ramanattam (geste de Rama), forme première de Kathakali, s'opposant au théâtre de temple Krisnanattam (geste de Krisna), donna naissance à un genre de poésie dramatique, ou Attakatha, en langue populaire malayalam, conçu à partir d'épisodes spectaculaires de la littérature sacrée et épique. Spécialement destinés au théâtre, ces textes engendrèrent des personnages archétypes, lesquels, par le déploiement de moyens plastiques et corporels, recréent l'univers démesuré de la mythologie. Alors que le Krisnanattam était en grande partie masqué, le Kathakali développa un art savant du maquillage en relief dont le système ornemental associé à la symbolique des couleurs (vert: vertu; rouge: égocentrisme; noir : primarité) tend à rehausser les traits psychiques des personnages sans entraver la mobilité de la musculature faciale.

Le spectacle est toujours donné de la nuit tombante au petit matin, à l'intérieur ou à proximité d'un temple, dans une riche demeure ou en plein air sur une aire de jeu spécialement aménagée et éclairée d'une haute lampe à huile de bronze (aujourd'hui renforcée de projecteurs). Le jeu linéaire, circulaire ou statique du Kathakali se satisfait d'un espace limité ( $6 \mathrm{~m} / 4 \mathrm{~m}$ environ); toutefois, lors de poursuites, combats, processions, les acteurs investissent les rangs des spectateurs. Le spectacle accompagne les festivités religieuses et/ou populaires, ou simplement familiales, et peut revêtir, selon les circonstances, un caractère propitiatoire. Une scène d'amour introduit généralement l'intrigue qui opposera les Asuras (titans / démons) aux Devas (déités) ; conflits, rivalités, relations amoureuses et combats sans merci sont à l'image du monde des humains. L'élément humoristique, comique (pouvant friser la caricature), reste le propre de certains types, principale- 


\section{MUSIQUES}

ment des Tatis, Karis et des gens du commun. Art de possession et de l'imaginaire mêlant raffinement et réalisme, le Kathakali est souvent mis en parallèle avec le "théâtre de la cruauté" d'Artaud par l'exaltation des sentiments et le paroxysme des situations où alternent dévotion, haine exacerbée, passion, vindicte, héroïsme, barbarie sanguinaire des châtiments laissant toutefois place à la rédemption. "

Le succès remporté au Théâtre des Nations inscrit Milena Salvini parmi les spécialistes du spectacle dansé tel qu'il se pratique dans tout le sous-continent indien. Elle sera alors chargée de différentes missions en Inde et en Indonésie, par l'Unesco et par le ministère de la Culture. Parallèlement, elle commence à enseigner dès le début des années 1970. "À la fois art martial, théâtre et art de vivre, le Khathakali me semble une excellente pratique pour les jeunes gens, dit-elle. J'y ai consacré un ouvrage, L'Histoire fabuleuse du théatre Khathakali à travers le Ramayana. C'est une approche que je souhaitais facile, essentiellement destinée la jeunesse. »

Alors qu'elle recherche un lieu fixe où pouvoir donner régulièrement ses cours de Kathakali, Roger Filipuzzi, le mari de Milena, découvre un atelier rue Wurtz dans le $13^{\mathrm{e}}$ arrondissement de Paris. Architecte dans l'âme, il transformera cette quasi ruine en un lieu agréable et convivial. " Le Centre Mandapa est la conception de mon mari, explique Milena Salvini. Il a cuuvé à l'acoustique du lieu. D'ailleurs, l'un des enregistrements qu'ily a réalisé, 24 Chants du Gita Govinda (Auvidis) a obtenu le Grand Prix du Disque en 1992. À nous deux, nous nous sommes partagé la tâche de développer le centre. » Roger Filipuzzi y réalise des enregistrements discographiques pour les labels Arion, Auvidis et Ocora. Milena Salvini crée l'une des premières écoles de danse indienne françaises, dont la réputation de sérieux s'est renforcée avec le temps. Aujourd'hui, entre les cours réguliers - principalement de Bharata Natyam et de Kathak - et les stages ponctuels, elle accueille chaque saison entre 100 et 150 élèves. La petite salle de spectacle du Centre Mandapa peut accueillir une centaine de spectateurs. Sa programmation est principalement consacrée aux petites compagnies de danse, de conte et à des ensembles de musiques du monde. Environ 150 spectacles sont présentés par saison.

"Dès l'origine de la programmation, nous avons présenté des musiques du monde, qu'elles viennent d'Afrique, d'Europe de l'Est, d'Indonésie, de Chine ou du Japon, dit Milena Salvini. Nous voulions faire découvrir des musiques rares, des artistes dont c'était souvent la première scène en France. Nous présentons de la musique seule, mais aussi accompagnée de danses, de paroles contées. Ne dit-on pas en Inde que la musique est l'art maitre que l'on retrouve dans toutes les formes d'art? " En 40 ans d'existence, le Centre Mandapa a servi de tremplin à de nombreux musiciens et danseurs. Parmi les artistes de musiques du monde qui y ont fait leurs premières armes avant d'accéder à une reconnaissance internationale, on peut citer l'Iranien Djamchid Chemirani, l'Ougandais Geoffrey Oryema, l'Algérienne Houria Aïchi ou la Chinoise Liu Fang. "Beaucoup d'artistes d'Inde qui se font remarquer dans notre salle sont programmés l'année suivante au Musée Guimet, dit la directrice du Mandapa. Nous entretenons aussi d'étroites relations avec le Théâtre du Soleil. »

Sa modestie l'empêche de rappeler ses initiatives pionnières. En 1985, elle et son mari organisent au Théâtre de l'Odéon Les 24 Heures du raga, événement auquel participèrent de prestigieux artistes venus de l'Inde du Nord, dont les Frères Dagar, maitres du chant dhrupad, et le magicien de la flûte bansuri Hariprasad Chaurasia. Ses collaborations avec Ariane Mnouchkine s'inscrivent aussi à l'occasion du changement de millénaire sur ce principe, à chaque raga correspondant une heure du jour ou de la nuit. En 2011, leurs passions partagées pour l'Inde s'unissent à nouveau 
Kathakali, photos extraites du film de M. Salvini et R. Filipuzzi, réalisé par Jacques Oger, L'Épopée du Mahabharata, tourné en décors naturels $₫$ D.R.

dans la mise en place d'une Grande nuit carnatique, dédiée cette fois au style de l'Inde du Sud. "Ariane Mnouchkine, nous a aussi permis de programmer des nuits entières de Kathakali. Et nous sommes en train de préparer une Nuit du Kûtiyattam à la Cartoucherie de Vincennes aux alentours de la Toussaint 2016."

\section{Un Art Intemporel}

"De même que nombre de découvertes sont souvent le fruit du hasard, c'est grâce à la remise à jour, au début du siècle, d'un ensemble de manuscrits sur feuilles de palmier révélant treize pièces du dramaturge Bhasa (IIe-III siècles) que l'intérêt se réveilla pour le théâtre sanskrit et son mode de représentation. Seul témoignage demeuré vivant d'un art de la scène vieux de près de deux mille ans, le Kûtiyattam allait peu à peu sortir de l'oubli. Sa redécouverte s'opéra tout d'abord au Kerala, son lieu d'origine, où il avait été miraculeusement préservé tout en restant ignoré des autres régions. Représenté en de rares occasions et pour un public élitiste ayant seul droit d'accès à l'intérieur du temple, le Kûtiyattam avait gardé ses qualités de théâtre rituel et sacré. Intacts étaient restés ses secrets et ses codes que les experts ont depuis entrepris de déchiffrer, remontant à la source de traditions de l'acteur peut-être les plus complexes de tout l'art classique indien. Ce n'est qu'en 1972 qu'un festival officiel de Kûtiyattam réunissant les 


\section{MUSIQUES}

derniers maîtres et leurs disciples, fut organisé à New Delhi par la Sangeet Natak Akademi, portant ainsi cette redécouverte au niveau national. »

\section{Qu'est-ce que le Kûtiyattam?}

" À la fois drame rituel, théâtre épique, opéra sacré et pantomime bouffonne, le Kûtiyattam associe tous les modes d'expression. Les protagonistes du drame se différencient par le costume et la coiffe, l'ornementation, et par le maquillage et ses couleurs dominantes: caractères divins (dominante verte), caractères égocentriques (dominante rouge), caractères primaires (dominante noire). Les théâtres traditionnels du Kerala ont poussé à l'extrême l'art d'un maquillage en relief donnant l'apparence d'un masque subtilement articulé n'entravant pas la mobilité du visage. L'on traduit couramment le mot Kûtiyattam par "ensemble d'acteurs", ou "drame concertant" (kuti : ensemble, attam : action dansée). Kûtiyattam sous-entend également une pluralité de modes de jeu : masculin et féminin - corporel et vocal - physique et plastique. Par sa globalité et son extrême exigence, il est le plus proche des concepts énoncés dans le Natya Shastra (ouvrage de référence datant du début de l'ère chrétienne) puisqu'il implique la participation conjuguée des moyens physiques et psychiques. Par son ancienneté, le Kûtiyattam domine l'histoire des arts du spectacle du Kerala, seule région de l'Inde qui a préservé des théâtres classiques intégrant l'action groupée et le solo d'acteur. Ancêtre du Kathakali (XVII s iècle) et d'autres spectacles de même famille, le Kûtiyattam laissa son empreinte dans la gestuelle, l'art du maquillage et du costume."

Milena Salvini et son mari Roger Filipuzzi ont été très actifs dans les premiers travaux du programme de l'Unesco visant à la reconnaissance et à la préservation du patrimoine culturel imma- tériel. Leur film paru en 1994 sur l'art du Kûtiyattam était couronné d'un prix par l'organisation internationale. Et ils ont largement contribué à l'inscription de cet art, l'une des plus anciennes traditions encore pratiquée, sur la liste représentative du Patrimoine culturel immatériel de l'humanité, lors de la toute première commission le 18 mai 2001.

Soutenu par la Mairie de Paris et la Drac île-deFrance, le Centre Mandapa mène un travail de proximité exemplaire, notamment en direction des plus jeunes. Durant la saison 2014-2015, il a accueilli environ 5000 enfants, venus en matinée assister à des spectacles de conte, de pantomime, de marionnettes, de théâtre d'ombres, etc. "Nos partenaires sont des écoles maternelles et primaires, quelques collèges et des centres de loisirs, dit Milena Salvini. Nous choisissons des spectacles ludiques, ouvrant sur des cultures d'ailleurs (Afrique, Chine, Maghreb, Inde, etc.), ancrées dans des traditions. Et nous veillons à ce qu'il y ait un échange avec les enfants. Les artistes programmés vivent principalement en Île-de-France. Beaucoup d'entre eux sont immigrés et c'est notre vocation de les aider à faire connaître leurs cultures. »

\section{Spectacle :}

Nuit du Kûtiyattam à la Cartoucherie de Vincennes, 31 oct. et $1^{\text {er }}$ nov. 2016

\section{Bibliographie :}

L'Histoire fabuleuse du théâtre Khathakali à travers le Ramayana, Paris, éd. Jacqueline Renard, 1990.

\section{Centre Mandapa \\ 6 rue Wurtz - 75013 Paris \\ Tél. : 0145899900 \\ www.centre-mandapa.fr}

